\title{
Will COVID-19 Lead to a Resurgence of Vaccine-Preventable Diseases?
}

This article was published in the following Dove Press journal:

Infection and Drug Resistance

\author{
Asmita Priyadarshini Khatiwada (iD ${ }^{\prime}$ \\ Nistha Shrestha $\mathbb{I D}^{2}$ \\ Sunil Shrestha $\mathbb{D}^{\prime}$
}

'Department of Pharmaceutical and Health Service Research, Nepal Health Research and Innovation Foundation, Lalitpur, Nepal; ${ }^{2}$ Department of Biostatistics and Epidemiology, University of North Texas Health Science Center, Fort Worth, TX, USA
Correspondence: Asmita Priyadarshini Khatiwada

Department of Pharmaceutical and Health Service Research, Nepal Health Research and Innovation Foundation, Lalitpur, Nepal

Tel +977-980829|95।

Email asmitapriyadarshinikhatiwada@gmail. com
Abstract: The outbreak of coronavirus disease (COVID-19) has been affecting various sectors, including regular health services negatively. Vaccination, which saves the lives of 2-3 million populations of different age groups every year, is one of the most affected services by COVID-19. The routine vaccination services and outreach programs have been halted owing to lockdowns imposed on account of escalated cases of COVID-19 all over the world, affecting millions of lives at the stake of vaccine-preventable disease (VPD) henceforth. However, as it is becoming evident that we will have to live with COVID-19 for some time and many countries have lifted the lockdown, vaccination programs and campaigns have resumed with stringent preventive measures to be followed for COVID-19. These programs and campaigns have been trying to overlook and provide the missed vaccination during the imposed lockdown period and continue the other vaccination services to the public in the best possible ways. The collaborative approaches of various health-related organisations, governments and the healthcare workers are necessary to deliver the routine and mass vaccination services in an improvised manner.

Keywords: coronavirus disease, COVID-19, immunization, vaccine preventable disease

\section{Introduction}

Administration of a vaccine helps a person to fight against infection by making the person immune against it. ${ }^{1}$ Infection such as measles, pneumonia, polio, influenza, pertussis, diphtheria, tetanus, which are fatal yet preventable through the administration of appropriate vaccines, are referred to as the vaccine-preventable diseases (VPD). ${ }^{1}$

Vaccination is a cost-effective and globally endorsed public health intervention to prevent mortality and morbidity across the world population from the VPD. ${ }^{2,3}$ In the absence of vaccination, the lives of children and their communities are at risk. ${ }^{4}$ Every year, more than 1.5 million people die due to VPD worldwide. ${ }^{5}$ World Health Organization (WHO) reports that the prevention of around 2-3 million deaths in all age groups is due to routine vaccination every year. ${ }^{1}$ Despite globalised efforts, about 20 million infants worldwide did not receive three doses of DiphtheriaTetanus-Pertussis (DTP) containing vaccines due to a lack of access to services. These challenges are more predominant in low-and-middle-income countries (LMICs). ${ }^{3}$ Common vaccination drives are conducted routinely within the healthcare facilities, and various campaigns and outreach programs are also scheduled to reach the rural and underserved areas/regions to ensure that all the infants, children and vulnerable population are vaccinated. .,6 $^{4}$ 
However, with the spread of Coronavirus disease (COVID-19) throughout the globe, vaccination programs have been halted in many countries, thereby increasing the threat of reemergence of the diseases easily prevented through vaccines. ${ }^{7}$ COVID-19 was initially reported from Wuhan, China, in December 2019 and has now spread throughout the globe as a pandemic. ${ }^{8}$ As of July 7, 2020, more than 11 million cases of COVID-19, with over half a million deaths globally, have been reported to the World Health Organization (WHO). ${ }^{9}$ Presently, the priority action has been towards combating COVID-19 by prevention and management. With the widespread local transmission of COVID-19 pandemic, many sectors and their routine works have been affected and often neglected. ${ }^{10}$ One of the essential health services facing disruptions due to the ongoing pandemic is the vaccinations programs. Several components associated with vaccination services are supply chain system, cold chain, storage and handling system, staff training and capacity building, organisation, planning and supervision, and monitoring, surveillance and response systems; almost all of which were affected directly or indirectly amid COVID-19 pandemic. ${ }^{11,12}$

Since the suspension of mass vaccination campaigns, millions of children globally are at risk of missing essential vaccines $^{13}$, and at least 80 million children under the age of one are estimated to be at risk for VPD. ${ }^{14}$ Such disruption is likely to generate reemergence of other VPD presenting as collateral damage of COVID-19. Currently, sporadic outbreaks of measles have been observed in different parts of the world including Cambodia, Nepal, Pakistan, Bangladesh, Iraq, Nigeria; cholera in Cameroon, Yemen, Bangladesh; diphtheria in Pakistan, Bangladesh and Nepal, and the situation may be exaggerated if the disruption to vaccination continue. ${ }^{15-17}$ Therefore, this paper intends to explore the impact of COVID-19 on vaccination across the world.

\section{Vaccination Services During COVID-I 9}

In the wake of the ongoing pandemic, WHO suggested that the mass vaccination campaign may be conducted after careful consideration of the risks and benefits, the country capacity, and the level of COVID transmission; however, routine vaccination programs need to be continued. ${ }^{18}$ The changes in vaccination services during
COVID-19 can be observed in terms of access and availability of vaccination.

\section{Access to Vaccination Services}

Primary strategies taken by various countries to respond to COVID-19 vary widely and include restrictive lockdowns, social distancing, testing, and isolation. ${ }^{19}$ Such measures tend to restrict access to vaccination services, causing disruption. Additionally, the vaccination services are also disturbed because of the imbalance in vaccine supply chains worldwide, shortages of personal protective equipment to the healthcare workers in many LMICs, unavailability of health workers or mobilisation of healthcare providers to manage COVID-19 patients. ${ }^{12,20}$

WHO guidance specifies the need to weigh multiple factors, including the risks to benefits in conducting mass vaccination campaigns during this pandemic. ${ }^{18}$ Since mass vaccination campaigns attempt to reach remote areas which are generally not covered by routine services, a lot of unreached children get their vaccine doses through mass vaccination campaigns, mainly in LMICs. However, children all over the world are likely to miss the vaccination services in 2020 due to fear of infection transmission. ${ }^{21}$

An example of a reduced vaccination rate due to disruption by COVID-19 has been observed in the United States (US). The vaccination rates in the US have decreased since the declaration of the COVID-19 emergency period starting March 13, 2020. Furthermore, there was decrease in vaccine administration for measles and vaccine orders in the early week of March 2020. This reduction was prominent among children aged greater than 24 months. ${ }^{22}$ Moreover, the increasing vaccine hesitancy in the US has already contributed to 10-year high cases of measles with over 1200 cases in $2019 .^{23}$ Subsequently, disruption in vaccination services during the pandemic can lead to increased risk of VPDs, particularly, among unvaccinated children. The resurgence of the VPD can be burdensome for the health systems and economy around the world which is already affected by the COVID-19. Such infectious disease outbreaks might amplify the financial burden by increasing healthcare utilisation and exaggerating morbidity and mortality. ${ }^{24}$

The vaccination services in Italy were modified based on epidemiology of different regions of the country. In the epicentre of COVID-19, Lombardy, routine vaccination was suspended on February 24, 2020, and the service was reopened partially on March 11, 2020. The complete services of vaccination were recommended from April 14, 
2020. Also, the government has planned to track down and provide services with greater priority to all those children within the country who have missed their regular doses once the services will be resumed. ${ }^{25}$

Around 5 million children might have missed their routine vaccination in the month of March this year across different villages of India because of various reasons including COVID-19 among them. ${ }^{26}$ The organisations responsible for carrying out vaccinations in the remote areas of the country have been involved in tackling with COVID-19 pandemic, migrants' movement detection, and awareness programs on preventive measures for COVID19. As a result, there is lack of proper vaccination services within the country. Also, since vaccination programs are being provided only at medical colleges, district hospitals and primary health centers, the population in the rural areas are having problems to reach the vaccination centers because of lockdown. ${ }^{27}$

In Nepal, a national vaccination campaign has been halted in mid-way, and routine vaccinations were suspended for almost two months as the country was announced under lockdown from March 24, 2020. Since the cessation of scheduled vaccination services, multiple outbreaks of measles have been reported in different districts of Nepal emerged with at least two fatalities. ${ }^{28}$

\section{Availability of Vaccines and Healthcare Providers}

With the overwhelming expansion of COVID-19, the healthcare providers are more focused on containing the pandemic, often requiring all-hands-on deck approach. In LMICs, provider issues such as shortage of healthcare workers, closed vaccine clinics, suspension of vaccination services, and lack of personal protective equipment among health workers have been identified as barriers in vaccine delivery due to COVID-19. ${ }^{29}$ These might lead to an increase in numbers of unimmunised or partially immunised children in community.

The availability of a sufficient amount of vaccine at the national and sub-national levels is important to consider as the production and supply of vaccines have also been affected by COVID-19. ${ }^{15,30}$ The vaccine shortages have also been a consequence of COVID-19, especially in lowand middle-income countries because of the border and air travel restrictions amid the pandemic. ${ }^{16}$ The consignment of Japanese encephalitis, diphtheria, pertussis, and tetanus conjugate vaccines, which was supposed to come to Nepal in April-May, was halted because of the imposed lockdown in the country. ${ }^{31}$ Beside the actual shortages of the vaccine, the shortage has been seen due to "infodemic" as well. Being influenced by the emerging hypothesis indicating that $\mathrm{BCG}$ vaccines are associated with antiviral immune response and decreased morbidity and mortality due to COVID-19, people have been going for unnecessary BCG vaccinations which could further lead to a shortage of the vaccine for the ones who are in actual need of it. ${ }^{32}$

\section{Management Approaches}

In the United Kingdom (UK), despite the increased caseload of COVID-19, National Health Service (NHS) has recommended the continuation of routine vaccination for children. The exception to vaccination services is given to families with any member who is under isolation or are infected with COVID-19. ${ }^{33}$

Nigeria observed decline in vaccination services due to lack of awareness about the importance of vaccination, threat of COVID-19 infection and problems in transportation. However, the routine vaccination has continued currently with the involvement of the National Primary Healthcare Development Agency (NPHCDA) in collaboration with WHO. NPHCDA ensured public awareness about the COVID-19 infection and vaccination as well and made sure that the local healthcare providers are aware of the infection prevention and control measures with provisions of personal protective equipment (PPE) as well. ${ }^{34}$

Another country with increased outbreaks of measles is Brazil. $^{35}$ In response to the critical situation, the Pan American Health Organization (PAHO) is working on training healthcare professionals and making the community aware of the pandemic and vaccination. ${ }^{36}$

While many countries are focusing on training healthcare providers regarding vaccination in the pandemic, LMICs often struggle in the implementation of vaccination. In response to growing concerns of increased risk of outbreaks of VPD during the pandemic, the government of Nepal has endorsed an interim guideline for the reinitiation of vaccination and other essential healthcare services. ${ }^{37}$ All the provinces of the country were suggested to recommence the routine vaccination and regular vaccination campaigns through local health centers and clinics. Additionally, preventive measures, such as physical distancing should be adopted. ${ }^{37}$ However, the development and endorsement of the guideline do not guarantee the implementation of it, and collaborative approaches from each level of government (federal, provinces and local) are 
crucial for the vaccination programs to restart efficiently throughout the country.

The Ministry of Health of New Zealand put forward a guide for healthcare professionals regarding the maintenance of vaccination service during the Level 4 COVID-19 condition. Vaccination programs of measles and pertussis for infants, influenza and pertussis vaccination for pregnant women, and influenza vaccination to high-risk groups were prioritised, among others. However, non-urgent vaccinations were delayed and various measures for safe vaccination conduct were suggested to healthcare providers. Some suggested measures were allocation of different times in a day and different areas in the clinics for consult visits and sick visits, only single patient at a time, waiting area in separate rooms, changes in the location for vaccination, whenever feasible, so that patients do not have to go around the clinics and hospitals and easily get vaccinated outside the facility in cars directly. ${ }^{38}$ As New Zealand is one of the first countries to control the widespread transmission of COVID-19, it may be predicted that new guidelines provided by the government for immunisation might have been implemented effectively.

There have been increased cases of measles along with cases of COVID-19 in Cambodia since January 2020. Nevertheless, the government of Cambodia restarted the vaccination outreach programs with declining cases of COVID-19 in May 2020 to prevent the further occurrence of new cases of VPDs, including measles, rubella, and pertussis. The myths and misinformation related to COVID-19 were also rectified during the vaccination campaigns. ${ }^{39}$

The WHO and United Nations Children's Fund (UNICEF) are working to make sure that routine vaccination is continued wherever and whenever feasible around the world by providing new guidelines for strengthening vaccination program focused on infection prevention, transmission prevention and control measures. ${ }^{7,23,40,41}$

Many countries have lifted the lockdown by July 2020, which could have facilitated the vaccination programs and campaigns to carry on. ${ }^{42}$ However, these services should be conducted with great care, following strict measures to prevent the transmission of COVID-19, which makes it more challenging. Stakeholders like Centers for Disease Control and Prevention (CDC) have developed new guidelines, which are easily accessible, and are to be followed for vaccination in this ongoing pandemic. ${ }^{43}$ Reports have shown that most of the people in higher-income regions (Europe, America, Russia) are not sure about vaccine safety; thus, have vaccine hesitancy. Contrary to this, almost all the people in lower-income areas (South Asia, Eastern Africa) believe that vaccines are safe and are essential to save lives from different fatal diseases. ${ }^{44}$ In the presence of such differences in thoughts among people, the chance of vaccination of their children seems to be influenced, and the children might not be appropriately vaccinated. The panic among the people because of COVID-19 and the economic burden it has resulted, might also hinder the vaccination of the children and vulnerable populations around the world.

In LMICs, lockdown and social distancing were identified as major barriers to vaccination services in the initial duration of the pandemic. Furthermore, logistical barriers towards access to vaccines were noticed, which seem to be amplified with a lack of vision and clear action plan about moving forward. ${ }^{29}$ The ongoing pandemic was introduced as a novel and massive threat which led to creating much chaos and panic in the healthcare system across the world. Consequently, many countries have been looking up to WHO international guidelines as the resource towards safe continuation to regular vaccination. ${ }^{29}$ While it is sensible to follow international guidelines, targeted protocols and country-specific guidelines must be created by LMICs to move forward. Every country has different national and regional settings that have an impact on routine service delivery. Therefore, international guidelines can be used but must be tailored to the needs and challenges of LMICs to unify and make clear and implementable action plans.

\section{Conclusion}

The vaccination services have been tremendously affected by the ongoing COVID-19 pandemic, keeping millions of lives around the globe at risk for VPD. Many cases of outbreaks of VPD are already reported to WHO from different parts of the world making the immediate restoration of vaccination services in the best possible ways, a dire issue to consider by the relevant authorities. The focus should be from providing the necessary safety equipment in sufficient quantity to health workers, giving training to the health workers particularly about safety measures to be followed while dealing with patients, creating awareness among the public on the importance of the vaccination for children, to aiding the health institutions to make necessary changes in the infrastructure to safely cater the vaccination services in an institution and in mass campaigns. The international organisations dedicated to global health, authorities of governments and the healthcare professionals should work hand in hand to deliver the routine and mass vaccination services in an improvised 
manner amid this pandemic. LMICs, in particular, have to create a tailored approach towards routine vaccination that serves the needs and addresses the challenges to routine vaccine delivery.

\section{Abbreviations}

BCG, Bacillus Calmette-Guérin; CDC, Centers for Disease Control and Prevention; COVID-19, coronavirus disease-19; DTP, diphtheria, tetanus, polio; LMIC, low-middle income countries; NHS, National Health Service; NPHCDA, National Primary Healthcare Development Agency; PAHO, Pan American Health Organization; PPE, personal protective equipment; UK, United Kingdom; UNICEF, United Nations Children's Fund; VPD, vaccine preventable disease; WHO, World Health Organisation.

\section{Author Contributions}

All authors made substantial contributions to conception and design, acquisition of data and interpretation of data; took part in drafting the article or revising it critically for important intellectual content; agreed to submit to the current journal; gave final approval of the version to be published; and agree to be accountable for all aspects of the work.

\section{Funding}

This paper did not receive any funding.

\section{Disclosure}

The authors declare no conflict of interest.

\section{References}

1. World Health Organization (WHO). Immunization; 2020. Available from: https://www.who.int/topics/immunization/about/en/. Accessed 30 June 2020.

2. Andre FE, Booy R, Bock HL, et al. Vaccination greatly reduces disease, disability, death and inequity worldwide. Bull World Health Organ. 2008;86(2):140-146. doi:10.2471/BLT.07.040089

3. World Health Organization (WHO). Immunization coverage; 2019. Available from: https://www.who.int/news-room/fact-sheets/detail/ immunization-coverage. Accessed 30 June 2020.

4. United Nations Children's Fund (UNICEF). Immunization program; 2020. Available from: https://www.unicef.org/immunization. Accessed 30 June 2020

5. Children's Hospital of Philadelphia. Global immunization: worldwide disease incidence; 2020. Available from: https://www.chop.edu/cen ters-programs/vaccine-education-center/global-immunization/diseasesand-vaccines-world-view\#: :text=Immunizations\%20currently\%20pre vent $\% 202 \% 20$ million,vaccine $\% 2$ Dpreventable $\% 20$ diseases $\% 20$ each $\%$ 20year. Accessed 01 July 2020.

6. Government of Nepal, Ministry of Health and Population. National immunization program; 2020. Available from: https://www.mohp.gov. np/eng/program/child-health-services/nip. Accessed 01 July 2020.
7. United Nations (UN) News. Experts underscore COVID-19 threat to global progress on child immunization; 2020. Available from: https:// news.un.org/en/story/2020/05/1064772. Accessed 01 July 2020.

8. World Health Organization (WHO). Timeline of WHO's response to COVID-19; 2020. Available from: https://www.who.int/news-room /detail/29-06-2020-covidtimeline. Accessed 05 July 2020.

9. World Health Organization (WHO). WHO Coronavirus disease (COVID-19) dashboard; 2020. Available from: https://covid19.who. int/. Accessed 07 July 2020.

10. International Labor Organization (ILO). Sectoral impact, responses and recommendations; 2020. Available from: https://www.ilo.org/glo bal/topics/coronavirus/sectoral/lang-en/index.htm. Accessed 01 July 2020.

11. World Health Organization (WHO). The elements of a strong immunization programme - and why we need to invest in them. 2015. Available from: https://www.euro.who.int/_data/assets/pdf_file/0008/281528/ Elements-of-a-strong-imm-prgm.pdf. Accessed 29 November 2020.

12. Ismail S, Mounier-Jack S, Glassman A, Yadav P, Baker P, Chalkidou K. Maintaining essential services in the times of COVID-19: vaccination delivery in low- and middle-income countries. Center for global development; 2020. Available from: https://www.cgdev.org/blog/main taining-essential-services-time-covid-19-vaccination-delivery-low-andmiddle-income. Accessed 29 November 2020.

13. World Health Organization (WHO). Over 13 million children did not receive any vaccines at all even before COVID-19 disrupted global immunisation - UNICEF; 2020. Available from: https://www.unicef. org/press-releases/over-13-million-children-did-not-receive-anyvaccines-all-even-covid-19-disrupted. Accessed 2 December 2020.

14. World Health Organization (WHO). At least 80 million children under one at risk of diseases such as diphtheria, measles and polio as COVID-19 disrupts routine vaccination efforts, warn Gavi, WHO and UNICEF; 2020. Available from:https://www.who.int/news/ item/22-05-2020-at-least-80-million-children-under-one-at-risk-ofdiseases-such-as-diphtheria-measles-and-polio-as-covid-19-disrupts -routine-vaccination-efforts-warn-gavi-who-and-unicef. Accessed 2 December 2020.

15. United Nations Children's Fund (UNICEF). Disruption of childhood vaccination in South Asia poses an urgent threat to children's health UNICEF; 2020. Available from: https://www.unicef.org/pressreleases/disruption-childhood-vaccination-south-asia-poses-urgentthreat-childrens-health. Accessed 02 July 2020.

16. Gavi The Vaccine Alliance. COVID-19: massive impact on lower-income countries threatens more disease outbreaks; 2020. Available from: https://www.gavi.org/news/media-room/covid-19massive-impact-lower-income-countries-threatens-more-diseaseoutbreaks. Accessed 02 July 2020.

17. Hoffman J, Maclean R. Slowing the coronavirus is speeding the spread of other diseases. The New York times; 2020. Available from: https://www.nytimes.com/2020/06/14/health/coronavirusvaccines-measles.html. Accessed 03 July 2020.

18. Gavi The Vaccine Alliance. Can routine immunization be carried out safely during the COVID-19 pandemic?; 2020. Available from: https://www.gavi.org/vaccineswork/can-routine-immunisation-becarried-out-safely-during-covid-19-pandemic. Accessed 03 July 2020.

19. Cohen J, Kupferschmidt K. Countries test tactics in 'war' against COVID-19. Science. 2020;367(6484):1287-1288. doi:10.1126/ science.367.6484.1287

20. World Health Organization (WHO). WHO and UNICEF warn of a decline in vaccinations during COVID-19; 2020. Available from: https://www.who.int/news/item/15-07-2020-who-and-unicef-warn-of -a-decline-in-vaccinations-during-covid-19. Accessed 2 December 2020.

21. Center for strategic and international studies (CSIS). Strengthening routine immunizations and responding to Covid-19; 2020. Available from: https://www.csis.org/analysis/strengthening-routine-immuniza tions-and-responding-covid-19. Accessed 03 July 2020. 
22. Santoli JM, Lindley MC, DeSilva MB, et al. Effects of the COVID19 pandemic on routine pediatric vaccine ordering and administration - united States, 2020. Morb Mortal Wkly Rep. 2020;69 (19):591-593. doi:10.15585/mmwr.mm6919e2

23. Centers for Disease Control and Prevention (CDC). Measles (Rubeola); 2020. Available from: https://www.cdc.gov/measles/casesoutbreaks.html. Accessed 03 July 2020.

24. World Health Organization (WHO). Guiding principles for immunization activities during the COVID-19 pandemic; 2020. Available from: https://canvax.ca/sites/default/files/2020-04/WHO_Guiding\% 20 principles $\% 20$ for $\% 20$ immunization $\% 20$ activities $\% 20$ during $\%$ 20the $\% 20$ COVID-19\%20pandemic $\% 20 \% 28$ Interim $\% 20$ Guidance $\%$ 29_2020.pdf. Accessed 03 July 2020.

25. World Health Organization (WHO). Countries working to sustain population immunity to vaccine-preventable diseases during COVID-19 pandemic; 2020. Available from: https://www.euro.who. int/en/countries/italy/news/news/2020/4/countries-working-to-sustain -population-immunity-to-vaccine-preventable-diseases-during-covid -19-pandemic. Accessed 03 July 2020.

26. Das S. India's infant immunization drive comes to a halt over Covid19 fear. Business Standard; 2020. Available from: https://www.busi ness-standard.com/article/economy-policy/india-s-infantimmunisation-drive-comes-to-a-halt-over-covid-19-fear -120042201411_1.html. Accessed 04 July 2020.

27. Banerjee SK. Dealing with immunization during the COVID-19 pandemic: India's experience. European Pharmaceutical Review; 2020. Available from: https://www.europeanpharmaceuticalreview. com/article/119520/dealing-with-immunisation-during-the-covid-19pandemic-indias-experience/. Accessed 03 July 2020.

28. Poudel A. Measles outbreaks reported in five districts including in Kathmandu and Lalitpur in last one month. The Kathmandu post; 2020. Available from: https://kathmandupost.com/national/2020/04/ 29/measles-outbreaks-reported-in-five-districts-including-inkathmandu-and-lalitpur-in-last-one-month. Accessed 04 July 2020.

29. Saso A, Skirrow H, Kampmann B. Impact of COVID-19 on immunization services for maternal and infant vaccines: results of a Survey Conducted by Imprint-The Immunising Pregnant Women and Infants Network. Vaccines. 2020;8(3):556. doi:10.3390/vaccines 8030556

30. World Health Organization (WHO). Immunization in the context of COVID-19 pandemic; 2020. Available from: https://www.who.int/ publications/i/item/immunization-in-the-context-of-covid-19pandemic. Accessed 04 July 2020.

31. Poudel A. Diminishing vaccine stock becomes a cause for concern for health officials. The Kathmandu post; 2020. Available from: https://kath mandupost.com/health/2020/05/03/diminishing-vaccine-stock-becomes -a-cause-for-concern-for-health-officials. Accessed 04 July 2020.

32. Namkoong $H$, Horita $N$, Ebina-Shibuya R. Concern over a COVID-19-related BCG shortage. Int J Tuberc Lung Dis. 2020;24 (6):642-643. doi:10.5588/ijtld.20.0240

33. National Health Service (NHS). NHS urges public to get essential vaccines despite coronavirus outbreak; 2020. Available from: https:// www.england.nhs.uk/2020/05/nhs-urges-public-to-get-essential-vac cines-despite-coronavirus-outbreak/. Accessed 04 July 2020.
34. World Health Organization (WHO). Nigeria drives routine immunization amid COVID-19; 2020. Available from: https://www.afro.who. int/news/nigeria-drives-routine-immunization-amid-covid-19-0. Accessed 04 July 2020.

35. World Health Organization (WHO). Measles-Mexico; 2020. Available from: https://www.who.int/csr/don/24-April-2020-measlesmexico/en/. Accessed 04 July 2020.

36. Pan American Health Organization (PAHO). PAHO Director calls for continued immunization to avoid risk of other outbreaks during COVID-19 pandemic; 2020. Available from: https://www.paho.org/ en/node/70468. Accessed 05 July 2020.

37. United Nations Children's Fund (UNICEF). Resuming routine immunization; 2020. Available from: https://www.unicef.org/nepal/ stories/resuming-routine-immunization. Accessed 05 July 2020.

38. COVID-19 and immunizations - guidance for health providers [homepage on the Internet]. Health Navigator; 2020. Available form: https://www.healthnavigator.org.nz/health-a-z/c/covid-19-andimmunisations-guidance-for-health-providers/. Accessed 05 July 2020.

39. World Health Organization (WHO). Responding to a measles outbreak during the COVID-19 pandemic; 2020. Available from: https:// www.who.int/cambodia/news/feature-stories/detail/responding-to -a-measles-outbreak-during-the-covid-19-pandemic. Accessed 05 July 2020

40. World Health Organization (WHO). Guidance on routine immunization services during COVID-19 pandemic in the WHO European Region, 20 March 2020; 2020. Available from: https://www.euro. who.int/en/health-topics/health-emergencies/coronavirus-covid-19/ technical-guidance/2020/guidance-on-routine-immunization-servicesduring-covid-19-pandemic-in-the-who-european-region,-20-march -2020. Accessed 04 July 2020.

41. World Health Organization (WHO). WHO/UNICEF joint statement maintaining routine immunization services vital during the COVID19 pandemic; 2020. Available from: https://www.euro.who.int/en/ media-centre/events/events/2020/04/european-immunization-week $-2020 /$ statements/whounicef-joint-statement-maintaining-routineimmunization-services-vital-during-the-covid-19-pandemic. Accessed 04 July 2020.

42. Aljazeera. 'No time to take foot off pedal' - WHO: coronavirus live updates; 2020. Available from: https://www.aljazeera.com/news/ 2020/06/jakarta-opens-indonesia-battles-coronavirus-live-updates -200607235344888.html. Accessed 05 July 2020.

43. Centers for Disease Control and prevention (CDC). Immunization schedules; 2020. Available from: https://www.cdc.gov/vaccines/sche dules/hcp/schedule-changes.html. Accessed 05 July 2020.

44. Vaccines: RM Low trust in vaccination 'a global crisis'. BBC News; 2020. Available from: https://www.bbc.com/news/health-48512923. Accessed 06 July 2020.
Infection and Drug Resistance

\section{Publish your work in this journal}

Infection and Drug Resistance is an international, peer-reviewed openaccess journal that focuses on the optimal treatment of infection (bacterial, fungal and viral) and the development and institution of preventive strategies to minimize the development and spread of resistance. The journal is specifically concerned with the epidemiology of antibiotic resistance and the mechanisms of resistance development and diffusion in both hospitals and the community. The manuscript management system is completely online and includes a very quick and fair peerreview system, which is all easy to use. Visit http://www.dovepress.com/ testimonials.php to read real quotes from published authors. 